\title{
De la tentation selon l'écriture et la théologie médiévale et Renaissante
}

Jean-Marie Maguin

\section{(2) OpenEdition}

Journals

Édition électronique

URL : http://journals.openedition.org/shakespeare/1149

DOI : 10.4000/shakespeare.1149

ISSN : 2271-6424

Éditeur

Société Française Shakespeare

Édition imprimée

Date de publication : 1 novembre 1997

Pagination : 123-138

Référence électronique

Jean-Marie Maguin, «De la tentation selon l'écriture et la théologie médiévale et Renaissante », Actes des congrès de la Société française Shakespeare [En ligne], 15 | 1997, mis en ligne le 01 janvier 2007, consulté le 03 mai 2019. URL : http://journals.openedition.org/shakespeare/1149 ; DOI : 10.4000/ shakespeare.1149 
D E L A T E N T A T I O N

S E L O N L'É C R I T U R E E T LA T H É O L O G I E M É D I É V A L E E ET R R N A IS S A N T E

Pour faire comprendre le mot «tentation», les dictionnaires séparent ce que la langue a uni sous le même mot; ce faisant ils séparent peut-être ce que Dieu, tel que l'homme peut en apprécier les faits et gestes et la volonté, a uni. Nous aurons largement l'occasion de revenir sur cette hypothèse subversive d'une certaine tradition théologique longuement et minutieusement construite, comme on renforce un barrage au point dont l'expérience et la science laissent présager qu'il est l'endroit faible de l'ouvrage.

L'O.E.D. retient comme premier sens : «1. The action of tempting or fact of being tempted, esp. to evil ; enticement, allurement, attraction» et ajoute «sometimes with more or less approach to senses 2 and $3 »$. Il cite d'emblée à titre d'illustration la tentation de Jésus au désert (Matt. iv, etc.) et la tentation des saints par les esprits malins, celle de saint Antoine par exemple.

Le sens 2 - dont le sens 1 se rapproche parfois - est défini comme : «The action or process of testing or proving; trial, test» et reçoit l'annotation: «Obs. or $\operatorname{arch}$. . Le sens 3 donne comme définition: «A severe or painful trial or experience; an affliction, a trial». Si le Concise Oxford Dictionary, dans sa septième édition (1982) note encore sous le verbe «tempt» en troisième sens : «(arch.) make trial of, try the resolution of, (God did tempt Abraham)», toute mention de cette alternative sémantique disparaît des dictionnaires plus récents, outils de consultation populaire. Le Petit Littré possède un article d'une charpente théologique orthodoxe: «Mouvement intérieur par lequel on est porté à des choses soit indifférentes, soit mauvaises 
[...]// Religion. Sollicitation au mal par la suggestion du diable ou par celle de la concupiscence. Induire en tentation//Spéc. La tentation d'Ève et d'Adam par le serpent». Il n'est pas fait mention du sens archaïque de «mise à l'épreuve». Le Petit Larousse est d'une théologie beaucoup plus sommaire, se contente de «Mouvement intérieur qui incite au mal : résister à la tentation // Désir : tentation de voyager». La simplification semble être une tentation puissante. L'article du Petit Robert reste inchangé dans l'édition de 1993 : «1. Ce qui porte à enfreindre une loi religieuse, morale ; impulsion qui pousse au péché, au mal, en éveillant le désir. La tentation, les tentations de la chair. Succomber à des tentations; résister à la tentation. Induire en tentation. 2 . Action du tentateur. La tentation de Jésus dans le désert. La tentation de saint Antoine. 3. Ce qui incite (à une action) en éveillant le désir. Tendance qui se manifeste alors => envie; désir: On avait envie de tout acheter, que de tentations! «Ne cède point à la tentation de briller, garde le silence» (Stendh.). FAM. Attachez-le au cas où il aurait la tentation de s'évader». Le Dictionnaire français-anglais de Randle Cotgrave, publié en 1611, est sémantiquement plus complet que nos dictionnaires populaires modernes. Il propose pour le mot «tentation» les traductions suivantes : «A temptation, tempting, proofe, essay, proving, assaying». Nous nous situons donc pour cet examen dans un champ notablement rétréci par le savoir moderne où l'idée que les dernières générations de rédacteurs de dictionnaires se font de ce que doit être le savoir moderne. Pour restaurer à ce champ ses dimensions d'origine, et donc à la réflexion sa portée primitive, il faut se tourner vers l'Écriture, les essais et gloses théologiques, et bien sûr les quelques dictionnaires spécialisés ou encyclopédies qui peuvent nous aider à y naviguer sans perte de temps excessive.

Dans l'Ancien Testament, la racine nissah est la plupart du temps rendue par prove, ainsi dans la Genèse 22.1 pouvons-nous lire God did prove Abraham (Bible de Genève ${ }^{1}$ ) mais le mot tempt/temptation est préféré pour les épisodes suivants : mise à l'épreuve par Dieu du caractère et des intentions de Pharaon (Deutéronome 4.34, 7.19, 29.3); pour la mise à l'épreuve de Dieu lui-même par Israël (Deutéronome 6.16; Exode 17.2 et 7 ; Nombres 14.22, Psaumes $78.18,41$ et 56) ${ }^{2}$. Dans le Nouveau Testament, le mot grec peirasmos est la plupart du temps rendu par tempt/temptation et exceptionnellement par trial, ainsi dès Matthieu 4.1 avec la tentation du Christ au désert. Tout le débat consistera à essayer d'établir si nous nous trouvons devant un phénomène d'équivocité, comme les théologiens nous encouragent à le penser, ou d'univocité déguisée.

Les incidences des mots try/trial,tempt/temptation dans l'Écriture sont liées à des situations d'apparente variété qui forment un catalogue intéressant. Il commence par ce qui va conditionner toute l'histoire et donc celle de la tentation: la tentation d'Ève et d'Adam par le serpent (Genèse 3). Il se 
poursuit par la mise à l'épreuve d'Abraham par Dieu lui réclamant le sacrifice de son fils unique Isaac, puis par le refus des enfants d'Israël de se soumettre à ce que Moïse leur décrit comme une mise à l'épreuve par Dieu tonnant sur le Mont Sinaï : c'est l'épisode des Tables de la Loi (Exode 20.20). Le catalogue se poursuit par l'abandon du roi Ezéchias par l'esprit de Dieu lors des pourparlers engagés avec les officiers des rois de Babylone : «God left him to try him, \& to knowe all that was in his heart» (2 Chroniques 32.31). Appel passionné du psalmiste à ce que sa foi soit éprouvée : «Prove me, ô Lord, and trye me : examine my reines, and mine heart» (Psaumes, 26.2). Triple tentation par le diable du Christ au désert (Matthieu 4). Tentation au centre de l'épisode du Jardin des Oliviers avec l'avertissement du Christ aux disciples : «Watch, and pray, that ye enter not into tentation : the sprit in dede is readie but the flesh is weake» (Matthieu 26.41) ; et avertissement qui sort de la bouche de celui qui vient de subir l'angoisse de l'épreuve qui s'annonce comme test de sa résolution divine dans sa nature humaine en contrepoint de la tentation du renoncement : $\ll \mathrm{O}$ my Father, if it be possible, let this cup passe from me : nevertheless, not as I wil, but as thou wilt» (Matthieu 26.39). Les notes marginales de la Bible de Genève révèlent des commentateurs peu empressés d'insister sur l'humanité pleine du Christ. Le commentaire des symptomes de l'angoisse : «began to wax sorrowful» (37) est «He feared not death of it self, but trembled for feare of Gods anger towards sinne, the burden wherof he bare for our sakes». Il s'agit donc d'une angoisse au deuxième degré, faite de sympathie pour les pécheurs. Le commentaire des paroles adressées à Pierre et aux deux fils de Zébédée : «My soule is verie heavie even unto the death» (38) reprend la même leçon : «For he saw Gods angre kindled towardes us». L'évocation de la possibilité que la coupe passe loin de lui attire cette précision : «That is the angre of God for mans sinnes. He knew wel what his Father had determined, and therefore was ready to obey but he prayeth as the faithful do in their troubles without respect of the eternal counsel of God». Plus tard, une fois sur la croix, lorsque le Christ est ironiquement invité à prouver sa divinité en se sauvant lui-même ou en en appelant Dieu pour le sauver de la torture $(« 42$. He saved others, but can he not save him self : if he be the King of Israel, let him now come downe from the crosse, and we wil beleve him. 43. He trusteth in God, let him deliver him now, if he wil have him : for he said I am the Sonne of God», le commentateur aborde enfin résolument le problème de la tentation : «This was a great tentation, to go about to take from him his truste in God, and so to bring him to despaire». Le commentaire des cris de désespoir qui suivent est un exercice plus périlleux : «46. And about the ninth houre Jesus cryed with a loude voyce, saying, Eli, Eli lama sabacthani ? that is My God, my God, why hast thou forsaken me». Le commentateur n'invoque pas ici la tentation (si tentation il y a, elle serait pour l'homme Jésus du même type que 
celle infligée par Dieu à Ézéchias : reflux spirituel plutôt qu'abandon physique) mais écrit : «Notwithstanding that he feeleth him self as it were wounded with Gods wrath and forsaken for our sinnes, yet he ceasseth not to put his confidence in God and call upon him : which is written to teache us in all afflictions to trust stil in God, be the assautes never so grievous to the flesh». L'angoisse est donc non le produit d'un quelconque doute sur la mission de Sauveur, voire sur sa nature de fils de Dieu que contestent les railleurs qui entourent la croix et interpellent Jésus, mais un sentiment d'aliénation vis à vis de Dieu par le poids du péché dont il s'est chargé.

L'Écriture et ses gloses autorisées ne sont pas les seules modalités de la réflexion sur la tentation. Les cycles des Mystères montrent au peuple la chute de Lucifer, la tentation et la chute d'Ève et d'Adam, Abraham et Isaac, la tentation du Christ au désert, la mort sur la croix pour ne prendre que les épisodes majeurs engageant un processus de tentation. Puis il y a des broderies intéressantes : la Second Shepherds' Play (Wakefield cycle) dans l'interlude de Mak montre les bergers - ceux de l'Évangile de Luc plongés pour les besoins de la cause dans une Angleterre hivernale contemporaine, en grand danger de succomber au péché d'idolâtrie en adorant le faux Christ que leur a fagoté le voleur de moutons. La principale manipulation par rapport à l'Ecriture consiste à montrer la chute de Lucifer, épisode qui n'est pas de la tradition scripturaire mais qui est né de l'invention d'une démonologie chrétienne tardive, bien représentée dans le corpus de textes du Pseudo-Denys l'Aréopagyte (Ve-VIe siècle) ${ }^{3}$. Les Mystères montrent un Lucifer qui est d'emblée dans le péché d'orgueil :

\section{Ah! Ah! That I am wonderous bright \\ Among yow shyning so cleare ! \\ Of all heaven I beare the light,}

Though God himself and he were here ${ }^{4}$.

Point de temps pour le doute et la vacillation puisque le temps est un concept absent du monde des anges. Le temps de jeu accordé à Lucifer est consacré à défier le Créateur et son pouvoir d'illumination : "All heaven shynes throughe my brightness, / For God himselfe shynes not so cleare» ${ }^{5}$, et à résister aux objurgations d'un certain nombre d'allégories positives. Cette chute est faite pour préparer la seconde, celle de l'homme, après qu'a été repéré et isolé le principe du mal. La pièce montée par les drapiers et qui montre la Création et Adam et Ève dans le cycle de Chester est très spécifique quant aux traits physiques sous lesquels le démon, qui déplore sa chute, compte aborder Ève : 
Feete as an adder, a maydens face ;

Her kindenes I will take ${ }^{6}$

Nous avons affaire ici à une créature inspirée par les monstres de la tradition classique, comme le remarque le présentateur du texte, les pieds appartiennent au serpent d'avant la malédiction divine (Genèse 3.14). Quant au visage féminin que l'on trouve aussi sous le pinceau de certains peintres et enlumineurs, il est le masque et la marque terrible de la discrimination qui voit une essentielle affinité entre mal et principe féminin et assoit l'autorité du patriarche sur ce mythe accusateur. Ève ne perçoit l'attrait physique du fruit défendu ( $\ll \mathrm{Ah}$, lord, this tree is fayre and bright, / Greene and semelye in my sighte» ${ }^{7}$ ) qu'après que le serpent lui a révélé le pouvoir qu'il confère : «Like goddes yow shall be» ${ }^{8}$. C'est donc l'orgueil qu'il incarne que l'esprit du mal souffle à Ève. Quand vient le tour d'Adam, le serpent n'a pas à intervenir, le prosélytisme trempe la chaîne de la tentation. Le cri de douleur, de repentir et la malédiction d'Eve sortent de la bouche d'Adam dans les trois vers qui suivent son consentement à transgresser la loi de Dieu («One morsell I will take») : «Out! Alas! What eales me ? / I am naked, well I see; Woman cursed must thou be» ${ }^{9}$. La rapidité avec laquelle s'est déroulée la tentation d'Adam n'empêchera pas d'expliquer, en ayant recours à saint Grégoire, la nature différente et triple de son péché. Là où Ève péchait seulement par orgueil, semble-t-il, Adam, lui, a péché par gourmandise («Glotony»), orgueil («vayne glorie») et - interprétation inattendue - par avarice («Avaryce»). Gourmandise du fruit, envie d'égaler la divinité, avarice pour entendre se prévaloir de distinguer le bien du mal. Dans la pièce sur la tentation du Chris jouée par les bouchers le Présentateur construit la perspective téléologique selon laquelle le nouvel Adam, le Christ, résistera lui à ces trois tentations. D'abord à la tentation née de l'appétit au terme de son jeûne :

Expositor: That Adam was tempted in Glotony,

I may well prove apertly,

When of the frute falsly

The Devill made hym to eate ${ }^{10}$.

Cette interprétation, ici, profite à Ève dont le rôle de relais dans la tentation est, pour une fois, complètement gommé. Le Christ refuse la suggestion du diable de changer les pierres en pain. Il résiste au péché d'orgueil lorsqu'il refuse de se jeter du faîte du Temple en forçant l'assistance des anges pour sa protection - il refuse donc de tenter Dieu. Il résiste enfin au péché d'avarice en refusant la couronne des royaumes terrestres que lui propose le diable contre allégeance à l'esprit du mal.

L'allégorisation des péchés entraîne l'auteur de l'Ancrene Riwle ${ }^{11}$ à faire apparaître des sous-catégories afin de mieux mettre en garde les nonnes contre 
la tentation. Les visages de cette dernière surgissent donc avec une variété que ne possèdent point les parades des péchés capitaux, au nombre de sept comme on le sait : avarice, colère, envie, gourmandise, luxure, orgueil, paresse - ici déclinés selon l'ordre alphabétique des noms français. Ce n'est pas là l'ordre théologique, repris par l'auteur du manuel de dévotion et assorti d'une symbolique animale :

But go with great caution for in this wilderness [crossed by the path leading to the heavenly Jerusalem] there are many evil beasts : the Lion of Jerusalem] there are many evil beasts : the Lion of
Pride, the Serpent of venomous Envy, the Unicorn of Wrath, the Bear of deadly Sloth, the Fox of Covetousness, the Sow of Gluttony, the Scorpion with its tail of stinging Lechery, that is lust. These listed in order are the Seven Deadly Sins ${ }^{12}$.

Le Lion d'Orgueil a beaucoup de petits parmi lesquels l'auteur en signale onze: Vainglory, Disdain, Hypocrisy, Presumption, Disobedience, Loquacity, Blasphemy, Impatience, Contumacy, Contention. Le onzième rejeton n'est pas nommé mais est décrit comme

fed with superstitions, with postures and gestures, such as carrying the head high, curving the neck haughtily, giving sidelong or scornful looks, blinking the eyes, pursing the mouth, making signs of derision with the hands or with the head, throwing one leg over the other, sitting or walking stiffly like one tied to a stake, giving affectionate looks to men, speaking like an innocent and affecting a lisp ${ }^{13}$.

Le passage suggère jusque dans la traduction les qualités d'observation satirique et la vivacité du propos. Avec ou sans nom, chacun d'entre nous a pour le meilleur ou pour le pire, rencontré ce lionceau d'Orgueil.

Le Serpent de venimeuse Envie a sept petits : «Ingratitude, Rancour or Odium, Displeasure at Another's Good, Being Glad when he [the other] Suffers Harm, Pointing out Faults, Backbiting, Reproaching people or Deriding them, Suspicion, Sowing of Enmity, of anger and of discord, Malevolent Silence». Emporté par la rhétorique dressée contre le péché et les visages de la tentation, les septs rejetons annoncés deviennent dix et étant désignés par des périphrases voient leurs traits singulièrement affinés ${ }^{14}$.

La Licorne de la Colère a six enfants (mais sept vont être dénombrés) : «Contention or Strife, Rage, Vilifying reproach, Cursing, Striking, Desire for Harm, Doing Evil out of Anger, or omitting to do good, or refusing to eat or 
drink, taking one's revenge in tears if one cannot do so in another way, shouting off one's head in angry cursing, or in other ways doing harm to oneself in both soul and body. This is homicide and self-murder». Ici la Licorne emprunte au Lion son dixième rejeton: Contention déjà nommé, prouvant ainsi que la plume du clerc courre plus vite que sa mémore. Par ailleurs, à la fin, le discours dépasse l'allégorie et saute de la nomination périphrastique au catalogue détaillé des tendances, faits et gestes coupables ${ }^{15}$.

L'Ours de lourde Paresse possède pour progéniture: «Torpor, Pusillanimity. Heaviness of Heart, Idleness, Grudging Heart, Deathly Grief, Negligence, Despair» (ce dernier étant signalé comme plus fatal que les autres) ${ }^{16}$.

La famille du Renard d'Envie compte: Treachery et Deceit, Theft, Rapine, Extortion, Unjust Exercise of Superior Strength, False Witness or Swearing, Concealed Simony, Excessive Taxation, Usury, Meanness, Unwillingness to Give or Lend, "and even sometimes Murder" 17

La Truie de la Gourmandise n'a que cinq enfants aux noms bien suggestifs : Too Early, Too Delicately, Too Voraciously, Too Much, Too often. Il est précisé qu'ils sont davantage portés sur les liquides que sur les solides ${ }^{18}$.

Le Scorpion de la Luxure affiche dans sa descendance : Fornication, Adultery, Loss of Virginity et Incest. L'expérience de la confession a développé chez l'auteur beaucoup de dextérité psychologique et celle-ci lui permet de nouvelles prouesses taxinomiques quand il détaille par exemple les modalités de la tentation incestueuse :

[it] takes place between those who are related, physically or spiritually, and it divides into many kinds : a foul desire towards the unclean action itself, together with the consent of reason; helping others towards it ; being aware of and witness to it ; pursuing it and trying to bring it about, by unchaste touch or by any other enticements, by frivolous laughter, wanton eyes, or any light behaviour, by gifts, by encouraging words, or with words of love, kissing, improper handlings, which may constitute mortal sin, taking pleasure in the time or place which may give rise to such things, and other occasions which he must of necessity avoid who would not fall disgracefully into the great mire ${ }^{19}$.

Ayant fait cette revue rapide des visages possibles de la tentation et du péché auquel le diable par vocation d'opposition autant que par esprit de vengeance, en souvenir de sa propre déconfiture - pour rappeler le spectre en 
ses deux extrêmes : le premier philosophique qui apparaît le plus souvent chez les théologiens, le second psychologique qui est mis en avant par les théologiens quelquefois et par les dramaturges toujours - il est temps, informés des voies du démon de revenir à celles de Dieu et à la tentation à laquelle il soumet l'homme. L'épisode de la tentation d'Abraham est certainement celui qui cause le choc le plus considérable et suscite le plus de commentaires ${ }^{20}$. Saint Augustin, dans le chapitre xxxii du livre XVI de $L a$ Cité de Dieu, traite «De l'obéissance et foy d'Abraham, par laquelle il a esté approuvé, au moyen de l'oblation de son fils qui devoit estre immolé...». L'ancien professeur de rhétorique qu'est saint Augustin fait dans ce chapitre flèche de tout bois, mais au total ses explications sont embarrassées. Il commence, de façon osée, par une invalidation de Dieu comme destinataire du sacrifice. «Abraham est tenté d'immoler son très cher fils Isaac, afin que sa devotieuse obédience fust approuvée, ayant à venir en connaissance aux siècles et non pas à Dieu ${ }^{21} »$. On retrouvera chez d'autres qui ont lu le docteur et père de l'église cet argument fondé sur la nécessité didactique - nécessité d'enseigner aux hommes qui vaut en somme tous les sacrifices. C'est pour notre bien qu'Abraham a levé le couteau sur son enfant. Le besoin humain est mis en avant-plan d'un quelconque besoin qu'aurait Dieu d'éprouver à ses propres yeux et à ses propres fins la foi d'Abraham. Le deuxième argument de saint Augustin entre dans le schéma téléologique. Le chrétien Augustin, qui voit dans tout l'épisode une préfiguration du sacrifice christique (Isaac a porté le bois du bûcher comme Jésus portera sa croix, le bélier aux cornes enchevêtrées dans le buisson annonce le Sauveur, la tête ceinte de la couronne d'épines), consiste à prêter à Abraham par anticipation, en quelque sorte, sa propre foi dans la résurrection du Christ : Abraham était persuadé que son fils ressusciterait aussitôt après le sacrifice. Saint Augustin passe le reste de son chapitre à débattre de ce problème difficile : comment Abraham pouvait-il savoir que ce ne serait pas Ismaël plutôt qu'Isaac qui serait privilégié puisque Ismaël est aussi annoncé comme le chef d'une grande nation et nommé semence d'Abraham. Sans que cela soit explicitement affirmé, il est assez clair que c'est la ressemblance entre la carrière du couple Yahvé-Isaac et du couple Dieu le Père-Dieu le Fils qui constitue la pierre de touche. Pouvait-elle stimuler la confiance d'Abraham au fort de sa tentation? En l'absence de don prophétique particulier ${ }^{22}$, on a bien l'impression que le savant chrétien crédite le patriarche juif de sa propre connaissance ex post facto pour raisons de convenance personnelle. Les épisodes fondateurs sont souvent violents, parfois iniques, celui-ci interpelle parce que semblant devoir jusqu'au dernier moment se ranger dans la deuxième catégorie, il a pour inspirateur déclaré Dieu lui-même.

Le problème de la compréhension au fond du processus de tentation issue 
de Dieu pour éprouver la foi de l'homme se noue dans le Notre Père, à la sixième pétition : «lead us not into temptation». Est-il besoin de rappeler qu'il s'agit là de la prière reçue du Sauveur dans le cadre du discours prononcé sur la montagne? Il n'y a pas de plus haute autorité pour le chrétien. La tradition catholique romaine, dans le but de faciliter la rencontre avec la tradition protestante a récemment adopté la formule «ne nous soumets pas à la tentation» en lieu et place de la formulation antérieure notamment moins rude ou ambiguë : «ne nous laissez pas succomber à la tentation». Bien des catholiques furent alors plongés dans un abîme de doute que l'usage leur avait dissimulé. Il faut signaler que les six grandes traductions anglaises de la Bible de 1380 à $1611^{23}$ - version catholique de Reims comprise - adoptent sans défaillance «lead us not into temptation». Or qu'est-ce que cela veut dire qu'un Dieu qui, par son fils - homme et Dieu à la fois - nous invite à demander à Dieu de ne pas nous soumettre à la tentation ? Il y a là un problème sérieux pour tous les théologiens persuadés que sans la tentation venue de Dieu la foi humaine n'est pas trempée. Il est presque de mauvais goût de souligner qu'en matière de tentation, qu'elle vienne du diable ou de Dieu, le Christ possède une expérience étendue. Voici donc posé de nouveau, de la bouche même du Sauveur le problème ardu de la relation agoniste qui subsiste éventuellement entre Dieu, l'homme, voire les forces du mal.

Les apôtres furent les premiers à réagir, dans des sens différents, à ce problème épineux. Saint Paul dans la Première Épître aux Corinthiens (Chap. 10) affirme :

13. There hath no tentation taken you, but such as apperteineth to man: and God is faithful, which wil nut her yil even give the issue wit temptation, that ye may be able to beare it.

Voici un argument fin et généreux mais qui a peu de chance de convaincre le chrétien moyen - angoissé comme le résistant qui avant une séance de torture ne sait pas s'il pourra tenir - surtout s'il connaît le très problématique livre de Job ${ }^{24}$

Écoutons saint Jacques dans le premier chapitre de son Épître générale :

2. My brethren, counte it exceeding joy, when ye fall into divers tentations,

3. Knowing that the trying of your faith bringeth forthe pacience.

4. And let pacience have her perfite worke, that ye may be perfite and entier, lacking nothing. 
12. Blessed is the man, that endureth tentation: for when he is tryed, he shal receive the crowne of life, which the Lord hathe promised to them that love him.

13. Let no man say when he is tempted, I am tempted of God: for God can not be tempted with evil, ne[i]ther tempteth he any man.

14. But everie man is tempted, when he is drawne

away by his owne concupiscence, and is entised.

15. Then when lust hathe conceived, it bringeth forth sinne, and sinne when it is finished, bringeth forthe death.

16. Erre not, my deare brethren.

Saint Jacques utilise une astuce rhétorique classique en abordant ses lecteurs avec un paradoxe qu'il va s'efforcer de démontrer. Cette déconstruction des positions pressenties chez les destinataires partage avec la lecon de Paul la conviction que la tentation fait partie de la nature, de la condition humaine, mais la ressemblance s'arrête là. Saint Jacques ne saurait pour rien au monde admettre le moindre lien entre Dieu et le mal. Saint Paul, avec son raisonnement numériste sur l'harmonie entre les forces offensives et défensives, esquive le problème.

Les commentaires sur le Notre Père qui se succèdent au XVIe et au XVIIe siècle vont d'un simple recto grossièrement imprimé à des opuscules développant chaque mot clé ou chaque proposition de ce texte. Pour ce qui est du passage qui nous intéresse, le but des commentateurs est triple : détailler les modalités de la tentation et ses effets sur la créature humaine, établir son origine, faire le départ entre la tentation du péché et la mise à l'épreuve de la foi. Sur un feuillet imprimé en 1627, que nous citons comme représentatif de l'ambition la plus modeste, «And lead us not into temptation» est assorti de quatre épithètes dressant autant de catégories de tentations et de types de souffrance en résultant : «temptation sudden which dismaieth», «temptation importunate which wearieth», «temptation deceitfull which circumventeth», et enfin «temptation violent which overthroweth». Ces quatre modalités se répartissent en deux grandes classes fondées sur le temps (soudaineté, caractère répétitif), et sur l'intensité du phénomène (procédant par la ruse ou se déclarant violemment). Les commentaires plus amples s'accordent pour voir trois origines à la tentation du péché ${ }^{25}$ : la première est interne à la nature humaine, c'est une tentation proprio motu et sa cause est la fragilité charnelle attestée par la concupiscence dont le Christ rappelle les dangers aux apôtres à Ghetsémani. La deuxième est externe et vient du monde. La troisième est l'œuvre du diable. Mais comme il ne faut pas donner de clarté illusoire à la victime d'un phénomène complexe, douloureux et dangereux, non plus qu'à 
ceux qui ont vocation de l'assister du point de vue spirituel, on remarque aussitôt que le diable est parfaitement au courant des failles de la nature humaine et sait se servir de la concupiscence comme d'un ressort pour conduire la foi à se trahir. C'est, dans la tentation au désert, la suggestion de transformer les pierres en pain et l'offre de régner sur les puissances du monde ${ }^{26}$.

Quant à la formulation même de la requête «lead us not into temptation», elle arrête tous les commentateurs. Certains avouent simplement leur perplexité, ainsi dans ce traité de 1579 peut-on lire :

It seemeth strange that God shuld lead into temptation. It is strange, I confesse, to mans reason, but Gods doing is above our reason. His judgements are alwayes just, though they be often secrete : and God then leadeth intoo temptation, when he punisheth one sinne with another : and when by taking away his sprite, and ditection he leaveth man to himself ${ }^{27}$.

L'exégète marque ici clairement la limite de l'exégèse. Un commentateur anonyme cherche à se sortir de la difficulté d'interprétation en adoptant une forme littéraire propre à couper court à toute discussion. C'est The Pater Noster spoken of the sinner: God answerynge him at every peticyon (STC 16818. $8^{\circ}$. T. Godfray, London, s.d.) :

\section{The synner}

Weake ar we o father / and feble / and the temptacyon grat and manyfolde in the flessh and in the worlde. Kepe us father with thy myghtye power / and let us nat fall in to temptatyon and synne agayne: but gyve us grace that we may abyde standinge / and fyght manfully unto the ende / for withoute thy grace we can do nothynge.

\section{IJ God}

I am rightuous / and right is my judgement / and therefore synne may na be unpunysshed / ye / and ye must suffre evyl and affliction / and as touching that ye have temptatyon therby / that is your synnes fauute only / which compelleth me therunto / to kyll it to heale you. for sinne can with none other medycyne be drawen out of you / but thorowe adversyte and sufferynge of evyll.

Le moins que l'on puisse dire est que le fruit de cette prosopopée est 
amer; et l'on retrouve ici la notion homéopathique du mal puni par le mal. Contrairement au domaine de la médecine, on ne doit pas craindre sans ce cas que le remède ne tue le malade, puisque la mort peut être le début du pardon et de la récompense.

Les traités les plus subtils se concentrent sur l'interprétation qu'il faut donner de «lead». A familiar and christian instruction upon the Lordes prayer de 1582, traduit du français et sortant de l'atelier de l'imprimeur-libraire huguenot Thomas Vautrollier (STC 16814. $12^{\circ}$ tr. by G. C[apelin]) se range dans cette catégorie. Le commentateur commence par affirmer à propos de la sixième requête : «The meaning is that we pray that no temptation overcome us». Le point de départ de la glose est donc trouvé dans l'avertissement du Christ aux apôtres à Ghetsémani (Matthieu 26.41, passage cité un peu plus loin parmi d'autres tirés de l'Écriture). Le commentateur évoque ensuite la théorie de saint Jacques sur le bienfait de la tentation comme mise à l'épreuve et le rappel que Dieu ne peut conduire au mal (Jacques 1.13). Il conclut : «To be led into tentation, is to be overcome and vanquished of tentation: as we are when we consent to the same, to be turned from God according unto the purpose and intention of the Devill». On note que l'argument se fonde sur la mesure ou l'intensité de la tentation, reprenant implicitement l'argument de saint Paul dans la Première épître aux Corinthiens (10.13), également citée parmi les passages à méditer. Il ne reste plus qu'à déclarer que la valeur de X, entendons le verbe «lead», est «trop fort pour nous» pour que l'on puisse alors instantanément prouver que la sixième requête veut dire «ne nous laisse pas succomber à Satan et nous détourner de toi». Il s'agit au total d'un texte habile et qui fait état des principales données, apparemment contradictoires, que fournit l'Écriture sur le problème de la tentation.

Le commentaire le plus complet et le plus incisif nous vient de William Perkins (1558-1602) ${ }^{28}$, un des grands théologiens anglais calvinistes, issu de Christ College, Cambridge et vénéré au XVIIe siècle presque à l'égal de Calvin ou de Hooker, dit-on. Son traité, An Exposition of the Lords Prayer parut d'abord en 1592 (STC 19700. $8^{\circ}$. R. Bourne a. J. Porter) et devait connaître trois autres éditions jusqu'en 1605. Perkins structure son commentaire en cinq parties : Cohérence, Signification, Applications, Mœurs à déplorer, Grâces à demander. Il commence par remarquer que la sixième pétition pourrait paraître superflue à certains, assurés du pardon des péchés. Ce n'est qu'une façon provocatrice et habile d'introduire une observation sur l'absence de tentations. Les seuls qui ne souffrent d'aucune tentation, dit Perkins, sont ceux qui sont déjà entièrement gagnés au diable. Puis il lie la sixième pétition à la cinquième ( «Forgive us our debts as we forgive our debts») et remarque qu'elle est une conséquence logique : ayant déjà péché, l'homme entend ne pas retomber dans l'erreur et demande l'aide de Dieu pour y parvenir. Pour naturelle que paraisse cette observation, elle révèle que Perkins, à la différence 
des autres commentateurs ne perd pas de vue la cohérence d'ensemble du discours que la méthode suivie de la glose point par point fait éclater, créant ainsi des obscurités artificielles. Il lie ensuite «Lead us not into temptation» à la proposition suivante : «but deliver us from evill». Il ne s'agit pas de deux pétitions séparées (comme le croient les autres glossateurs mais d'une seule et même pétition: «Lead us not into temptation: how is that done ? by delivering us from evill». La tentation est ensuite fermement définie de la façon la plus orthodoxe :

Temptation is nothing els, but the enticement of the soule or heart, either by the corruption of mans nature or the allurements of the world, or the devill, to any sinne. James 1.15 God tempts no man : that is, God mooves no man to sinne.

«Lead us not» est ensuite défini comme signifiant :

Or carrie us not into temptation. To be led, is to be overcome of the temptation when it prevailes and wholly gets the victory : so as men tempted are brought to perdition. Then the meaning is this. When we are mooved or entised to sinne, Lorde keepe us that we be not overcome; and give thou an issuewith the temptation.

Nous avons déjà rencontré cet argument dix ans plus tôt, et il se termine par une reprise de la phraséologie de saint Paul (1 Cor. 10.13) «but will give the issue even with the temptation». Perkins a ensuite recours à l'hypophore pour régler le point le plus fondamental. Voici comment il pose la question à laquelle il entend apporter réponse : «Quest. God is just and cannot sinne but if he lead men into temptation shall he not be the author of sinne? Ans. Inddeede many fearing to charge God with sinne, read the words thus, Suffer us not to be ledde. But the text is very plaine, Lead, or carry us not». Perkins cite alors trois passages de l'Écriture (Exode $7.3 ; 2$ Samuel, 24.1;2 Thessaloniciens 2.11 évoquant des actions de Dieu pour enfoncer l'homme dans l'erreur, ainsi comme lorqu'il durcit le cœur de Pharaon. Ceci permet a théologien d'aborder enfin le cœur du problème : le mal n'est jamais absolu :

There is no action of man, or of the devill, absolutely evill, yet in some other way it is good: for we are not to thinke that as there is a maine or absolute good, so also there is a maine and absolute evill. Thus then temptation being an action, it is not in every respect evill : but in some good, in some 
evill. And so farre forth as it is good, the Lorde workes it: but as it is evill, he doth not worke it :

but willingly permits to be done by man and Satan.

Cette théorie, fondée sur l'asymétrie du bien et du mal - bien absolu, mais mal relatif - permet, in abstracto du moins, de comprendre le rapport entre Dieu, Satan et Job par exemple. En bon calviniste, William Perkins s'appuie sur cette réflexion quant au caractère inévitable du mal pour arguer de la nécessité de la grâce et condamner la théorie papiste du libre arbitre. Il entre hardiment dans l'évocation de cette essentielle imbrication du mal dans le bien par deux exempla qu'il vaut de citer :

One man kills another: the very mooving of the bodie in the doing of this villanie is of God : but the wickednesse of the action is from man and the devill. A man rides up on a lame horse, and stirres him : the rider is the cause of the motion, but the horse himselfe of the halting in the motion. So God is author of the action, but not of the evil of the action.

Voici le problème de la tentation fermement réinscrit au cœur de la triade Dieu, homme, diable, et de telle façon à nous laisser croire que l'homonymie singulière qui a servi de point de départ à cette réflexion ne recouvre pas une totale équivocité mais plutôt une équivocité relative. Les ingrédients de toute tentation sont les mêmes mais seulement comme le sont aussi les ingrédients du bon et du mauvais vin. Ces propos ne sont pas précisément de nature à calmer l'angoisse existentielle et métaphysique de l'homme-vigneron.

Jean-Marie MAGUIN

Centre d'Études et de Recherches sur la Renaissance Anglaise UPRES-A 5065 CNRS

Université Paul-Valéry - Montpellier III

\footnotetext{
${ }^{N}$ O T E S
} 
${ }^{1}$ Nous y empruntons toutes les citations par la suite.

2 Références empruntées à James Hastings, éd., Dictionary of the Bible, New York, Scribner's Sons, 1918.

${ }^{3}$ L'auteur explique l'origine des démons en déclarant qu'il s'agit d'anges qui ont trahi leur nature.

${ }^{4}$ Chester cycle, 1. Tanners : The Fall of Lucifer (19) in Peter Happé, éd., English Mystery Plays, Harmondsworth, Penguin Books, 1975, p. 54.

5 Ibid., (23), p. 55

${ }^{6}$ Ibid., 2. Drapers : (25), p. 70.

7 Ibid., (31), p. 71.

8 Ibid., (29), p. 71.

${ }^{9}$ Ibid., (32) et (33), p. 72.

${ }^{10}$ Ibid., 22 Butchers (22), p. 394.

${ }^{11}$ Début du XIIIe siècle, $c .1215$.

${ }^{12}$ The Ancrene Riwle, translated into modern English by M.B. Balu, Introduction by Gerard Sitwell, London, Burns and Oates, 1955, Part IV : Temptations, p. 86.

${ }_{13}$ Ibid., p. 88.

${ }^{14}$ Ibid., p. 88-89.

15 Ibid., p. 89.

${ }^{16}$ Ibid., p. 90

17 Ibid., p. 90.

18 Ibid., p. 91.

${ }^{19}$ Ibid., p. 91.

${ }^{20}$ Nous pouvons attester la violence du choc ressenti par le spectateur dans la mise en scène sobre, pour ne pas dire austère, de Katie Mitchell pour la Royal Shakespeare Company à Stratfordupon-Avon (The Creation, The Other Place, saison 1996-1997). Dieu joué comme un personnage bonasse, goguenard vient s'accroupir presque à portée de main d'Abraham qui se livre aux derniers préparatifs, lie Isaac et sort le couteau. Dieu s'allonge alors sur le dos, bras en croix - insoutenable sommeil d'indifférence ou, par la position, prolepse du Fils en croix, ou encore discipline visant à canaliser la volonté à travers l'ange qui vient d'apparaître pour prévenir le sacrifice ? Benjamin Britten témoigne de son aversion pour cet épisode de l'Écriture en 
choisissant, dans une cantate impressionnante, de faire du personnage d'Abraham, une sorte de fanatique sanguinaire, un nouvel Hérode courant l'Europe pour exiger les premiers nés de toutes les familles, ou si l'on veut un Hitler car l'œuvre est écrite pendant la deuxième guerre mondiale alors que, justement, la descendance d'Abraham subit l'holocauste.

${ }^{21}$ Saint Augustin, De la Cité de Dieu, [traduit] par Gentian Hervet..., Paris, Nicolas Chesneau, 1579, p. 108.

${ }^{22}$ C'est au sens général d'homme ayant des relations privilégiées avec Dieu qu'Abraham est qualifié de «prophète» par Dieu s'adressant en songe à Abimélek, Genèse 20.7 (Bible de Jérusalem).

${ }^{23}$ Wyclif, 1380 ; Tyndale, 1534 ; Cranmer, 1539 ; Genève, 1537 ; Reims, 1582 ; version dite «autorisée», 1611.

${ }^{24}$ Livre fondamental pour cette réflexion, mais trop délicat pour être abordé justement dans le cadre d'une étude restreinte comme celle-ci. Le personnage de Satan (Chap. 1 et 2), qui veut dire 'adversaire' en hébreu, sa relation avec Dieu, son rôle et sa motivation dans l'histoire de la tentation de Job - en a-t-il une indépendante de celle de Dieu ? - , l'accumulation des malheurs, l'issue narrative «lisse» à défaut d'être réputée heureuse au bout d'une chaîne de pertes humaines irréversibles, sont autant de problèmes extraordinairement difficiles.

${ }^{25} \ll$ We are assauted with three manners of temptacions / the flesshe / the worlde /and the devyll». STC 16821.3 The pater noster, ye crede \& the commaundments in englysh. $8^{\circ}$. [R. Redman ? c. 1538].

${ }^{26} \mathrm{La}$ tentation au désert est rapportée seulement dans les évangiles synoptiques et de façon différente. Matthieu (4.1-11) adopte l'ordre suivant : 1) tentation du corps en jouant sur la faim ; 2) tentation de l'esprit jouant sur le désir de mettre Dieu à l'épreuve ; 3) tentation du corps en jouant sur l'attrait des richesses de ce monde. Luc (4.1-13) donne un compte rendu différent en suivant un classement 1, 3,2 qui épuise les tentations du corps avant de présenter celle de l'esprit. Luc d'une phrase de conclusion qui n'existe pas chez Matthieu témoigne d'une conscience plus nette de la stratégie utilisée par le diable pour 
dévoyer Jésus : «13. And when the devil had ended all the tentacion, he departed from him for a season» («Ayant ainsi épuisé toutes les formes de la tentation, le diable s'éloigna de lui, pour revenir au temps marqué», Bible de Jérusalem). Marc (1.12-13) se contente de mentionner que Jésus au désert fut tenté par le diable sans donner plus de détail.

${ }^{27}$ STC 1684.5: A godly and short treatise upon the Lordes prayer, the xxii articles of the Christian faith, and the ten commaundments. $8^{\circ}$. T. Dawson f. G. Bishop, 1580. Ent. 30 jn. 1579.

Son ouvrage le plus connu est Armilla aurea (1590), traité de théologie, qui en latin ou dans une version anglaise ( $A$ golden chaine) devait connaître douze éditions jusqu'en 1612. Sur William Perkins on consultera, entre autres, Christopher Haigh, English Reformations: Religion, Politics, and Society under the Tudors, Oxford, Clarendon Press, 1993. 